\title{
Determination of Tadalafil in pharmaceutical preparation of combination therapy with complex of vitamins and minerals by HPLC-UV detector.
}

\section{Ana Clara Muniz*, Paulo Pires da Rosa.}

\begin{abstract}
The method of determining Tadalafil in a mixture with a complex of vitamins and minerals by HPLC was verified to be linear, precise, selective and accurate with the use of WATERS Nova-Pack $B$ C8 $(3.9 \times 150) \mathrm{mm} ; 4 \mu \mathrm{m}$ column. The mobile phase used was water:acetonitrile:trifluoroacetic acid (60:40:0.1) at the flow rate of $1 \mathrm{~mL} / \mathrm{min}$ with UV detection at $285 \mathrm{~nm}$ at ambient temperature. Linearity was determined between 0.08 and $0.11 \mathrm{mg} / \mathrm{mL}$ with a correlation factor of 0.998. Standard deviations of performed tests of precision and accuracy were insignificant and components of the matrix did not interfere in the retention time of Tadalafil, the method is selective.
\end{abstract}

\section{Key words:}

Tadalafil, vitamins and minerals, combination therapy.

\section{Introduction}

Erectile dysfunction is described as the impossibility of maintain erection long enough to achieve a satisfactory sexual activity ${ }^{1}$. Nitric oxide is atypically found in low concentrations in cavernous corpus cells and actual therapy aims at increasing these levels by accumulation of GMPc. This is achieved by inhibition of the family of phosphodiesterases with the use of, for example, Tadalafil. The major toxicity inherent to this type of medicines is the hemodynamic alteration that normally cause hypotension ${ }^{2}$. Due to the cardiovascular risks involved in high punctual doses $(20 \mathrm{mg})$ in the elderlies, such administrations are not safe enough and diary doses $(5 \mathrm{mg}$ ) come as a solution. The present research has as line base the hypotheses that the combination therapy of Tadalafil with a complex of vitamins and minerals (V\&M) can be used as a feasible treatment for erectile dysfunction. The main goal is to study the compatibility of Tadalafil with the complex of V\&M as the transporter for dairy doses. To achieve this goal analyses of such mixture were developed by using HPLC-UV detector according to the American Pharmacopoeia ${ }^{3}$.

\section{Results and Discussion}

For the appropriate analyses was used acetonitrile-water $(1: 1)$ as solvent. The mobile phase was acetonitrile:water:trifluoroacetic acid (40:60:0.1) with an isocratic flow of $1 \mathrm{~mL} / \mathrm{min}$. The chosen column was very similar to the one specified (L7 $4.6 \mathrm{~mm} \times 15 \mathrm{~cm} ; 3.5 \mu \mathrm{m}$ ): WATERS Nova-Pack $®$ C8 $3.9 \mathrm{~mm} \times 15 \mathrm{~cm} ; 4 \mu \mathrm{m}$ series T50860P18. The HPLC Waters has a bomb Code 515 série \#D01515 315M; Detector $2487 \lambda$ dual absorbance; and Autosampler 717plus. All described studies were taken place at ambient temperature. Injections had $10 \mu \mathrm{L}$ of volume and detection was made at $285 \mathrm{~nm}$. The procedure for the samples was initiated by the grounding until fine powder of a commercialized complex of V\&M. The concentration of Tadalafil in the mixture was calculated to match the daily requirements of $5 \mathrm{mg}$ in two doses of capsules size 00 accompanied with the amount necessary of a daily dose of the complex of V\&M as specified in the drug leaflet. The calibration curve $(n=4)$ was used to determine the concentration of the compound in the simulation of the pharmaceutical preparation and linearity of standard Tadalafil was between 0.08 and $0.11 \mathrm{mg} / \mathrm{mL}$ with a correlation coefficient of 0.998 . Analyses of precision were also carried out with no remarkable variation in the interday tests. Accuracy was also proved with insignificant values of standard deviation. Selectiveness was proved after no interference of the complex of V\&M was made in the retention time of Tadalafil.

Figure 1 - Chromatogram

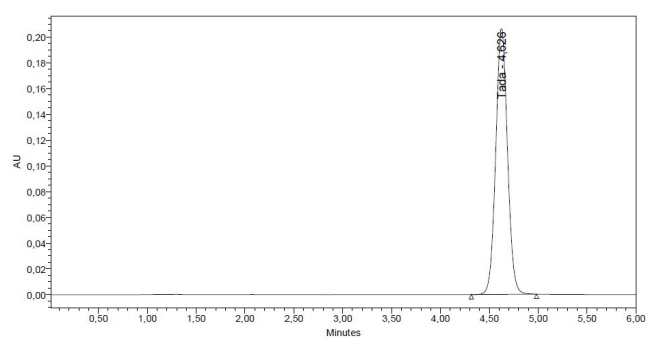

\section{Conclusions}

A chromatographic method to determine Tadalafil concentration in a matrix of the complex of V\&M was partially validated. Such matrix is reliable for the transport of Tadalafil in a concentration of $2.5 \mathrm{mg}$ in $0.617 \mathrm{~g}$ proved by the adequate quantitative determination of the active in the mixture. Further experiments will be performed to complete the validation of this method.

1.LUE, T. F. et al.. Summary of the Recommendations on Sexual Dysfunctions in Men, J. Sex Med, Vol. 1, No. 1, p.2-23, 2004.

2.GOODMAN, L. S. et al. Goodman \& Gilman's the pharmacological basis of therapeutics. New York, NY : McGraw-Hill, 2011.

3.The United States Pharmacopeia : the National Formulary. Rockville, MD. United States Pharmacopeial Convention, Vol. 3, p.5991-5995, 2016. 